\title{
Sozialstaatsentlastung durch mehr Familiensolidarität
}

\author{
Ursula Köbl
}

I. Öffentliche Familienförderung im Zentrum der Politik - Verstärkung privater Familiensolidarität am Rande

II. Monetäre Anerkennung für private Langzeitpflege und Kleinkindbetreuung 396

III. Ausbau der Familiensolidarität zugunsten von Kindern Überforderungstendenz bei „Patchworkfamilien“

IV. Unterhaltsbeziehungen zwischen erwachsenen Kindern und Eltern 401

V. Ambivalente Tendenzen in Bezug auf nicht (mehr) verheiratete Partner 403

VI. Normativ stabile Familiensolidarität im Erbrecht 407

VII. Schlussbemerkung: Unvermeidlichkeit von Zielkonflikten 411

\section{I. Öffentliche Familienförderung im Zentrum der Politik - Verstärkung privater Familiensolidarität am Rande}

Die Hochkonjunktur von Familienpolitik und Familienförderung sowie ihre ebenso starke und vielstimmige Begleitung durch die Sozialrechtswissenschaft ${ }^{1}$ lassen es nicht sinnvoll erscheinen, in dieser Perspektive verharrend, nun akribisch nach irgend disku-

* Frau stud. jur. Katrin Mysliewic und Herrn stud. jur. Martin Würthner danke ich vielmals für ihre Hilfe.

1 Repräsentativ BMFSFJ (Hrsg.), Familie zwischen Flexibilität und Verlässlichkeit, Siebter Familienbericht, BT-Drs. 16/1360; Tagungsband „Familie und Sozialleistungssystem“, SDSRV 57 (2008), mit Beiträgen von Fuchs, Maximilian, Hase, Friedhelm, Ruland, Franz, Kingreen, Thorsten, Münder, Johannes und Knickrehm, Sabine; Eichenhofer, Eberhard, Ehe und Familie im gegenwärtigen Sozialrecht, VSSR 2008, S. 85; ders., Ehe und Familie in der Sozialrechtsordnung, in: Behrends (Hrsg.), Gesetzgebung, Menschenbild und Sozialmodell im Familien- und Sozialrecht, Berlin 2008; Schwab, Dieter, Familie und Staat, FamRZ 2007, S. 1; Kingreen, Thorsten, Familie als Kategorie des Sozialrechts, JZ 2004, S. 938.

Schlaglichtartig wird die enorme Karriere der sozialstaatlichen Familienthematik deutlich an der erst zwei Jahrzehnte zurückliegenden Darstellung der Entwicklung in: Blüm, Norbert/Zacher, Hans F. (Hrsg.), 40 Jahre Sozialstaat Bundesrepublik Deutschland, 1989 (Zacher, Hans F., S. 48, 84, 100; Kaufmann, Franz Xaver, S. 547-560); Zacher, Hans F., Ehe und Familie in der Sozialrechtsordnung, in: Fiedler (Hrsg.), Verfassungsrecht und Völkerrecht, Gedächtnisschrift für Wilhelm Geck, Köln u. a. 1989, S. 955; ders., Abhandlungen zum Sozialrecht, Heidelberg 1993, S. 555. 
tablen Aussagen auf eben dem Feld zu suchen, das uns Eberhard Eichenhofer ${ }^{2}$ so weit ausgreifend, analytisch eindringlich und bezügereich dargestellt hat. Nur zwei Punkte, ein sozialpolitisch empirischer und ein sozialethischer, seien deshalb kurz angesprochen: zum einen das behauptete Motivationsgemenge für den neueren Aufschwung der Familienpolitik und zum anderen die angebliche Irrelevanz der genetischen Abstammung von Kindern im modernen Neben-, Nach- und Durcheinander familiärer Beziehungen ihrer genetischen Eltern und der sie versorgenden Personen. ${ }^{3}$

Wenn auch Juristen als solche sich wohlweislich vor einer näheren Faktenbeurteilung und -gewichtung hinsichtlich sozialpolitischer Triebkräfte zurückhalten sollten, so darf doch geäußert werden, was sich schon dem interessierten Laien aufdrängt und von vielen Fachleuten inzwischen ebenfalls offen ausgesprochen wird: In der neueren Phase zumindest der deutschen Familienpolitik wirkt sehr deutlich das bevölkerungspolitische Anliegen als Hauptantriebsfaktor. ${ }^{4}$ In erster Linie ist es doch wohl die für die gesamtgesellschaftliche Entwicklung 5 , längst nicht nur für die Altersversorgung ${ }^{6}$, besorgniserregende, ja bedrohliche Knappheit an Nachwuchs, die nicht nur zur tendenziell steigenden, unmittelbaren finanziellen Unterstützung der Eltern durch Sozialtransfers (und Steuerverschonungen) führt, sondern die auch, um die Vereinbarkeit von Erwerbstätigkeit und Familienarbeit zu fördern, den Ausbau der Betreuungsinfrastruktur ${ }^{7}$ vorantreibt. Die weithin erstrebte Erwerbstätigkeit der zunehmend gut ausgebildeten Frauen und deren hierdurch wie nie zuvor hohe Chancen zur nachhaltig volleigenständigen Existenzsicherung (abseits von familiärem Unterhalt und Sozialtransfers) wirken in dieselbe Richtung. ${ }^{8}$

2 Eichenhofer, Eberhard, Sozialleistungen und Familie, S. 349 ff. in diesem Band.

3 Ich möchte mich entschieden dagegen wenden, dass man diejenigen als engherzige, sozialethische Hinterwäldler diffamiert, die noch Gewissheit darüber haben wollen, ob die ihnen rechtlich und sozial zugeschriebenen Kinder oder Eltern auch ihre biologischen Kinder bzw. Eltern sind. Ein anerkennenswertes Interesse von Kindern insoweit dezidiert leugnend Ramm, Thilo, Ehelichkeitsanfechtung und Bundesverfassungsgericht, NJW 1989, S. 1594, 1595.

4 Vgl. Bertsch, Frank, Eine Familienpolitik für junge Familien, NDV 2004, S. 210; Schwab (Fußn. 1), FamRZ 2007, S. 1, 4; Leitner, Sigrid, „Paradigmenwechsel“ in der Familienpolitik usw., ZSR 54 (2008), S. 209, 216; GVG Informationsdienst, Nr. 318, Familienpolitik und soziale Sicherung in Europa, Köln, März 2007, darin Tiemann, Susanne, Einl. S. 4, und Meier-Gräwe, Uta, S. 6 f., anders wohl für Schweden: Andersson, Gunnar, S. 9 (hier primär auslösend: Gleichstellung der Geschlechter).

5 Repräsentativ Birg, Herwig, FAZ v. 28.6.2006, S. 43; ders., Demografische Zeitenwende, München 2004.

6 Wenngleich von daher die bis zur Jahrhundertwende andauernde Tabuisierung der Bevölkerungspolitik in der öffentlichen Meinung und im politischen Prozess ihr Ende fand. Treffend dazu Braun, Johann, Wahn und Wirklichkeit - Über die innere Verfassung der Bundesrepublik Deutschland, Tübingen 2008, S. 55 ff., 59 m. w. N.

7 Zuletzt das „Kinderförderungsgesetz“ (KiFöG), das ab 1.8.2013 den Anspruch auf einen Betreuungsplatz für jedes Kind zwischen eins und drei Jahren vorsieht (in § 16 Abs. 4 SGB VIII).

8 Schwab (Fußn. 1), FamRZ 2007, S. 5, mit krit. Blick auf die ausgeprägte Favorisierung der Doppelverdienerehe durch die aktuelle Gesetzgebung. 
In sozialpolitischer Hinsicht kann mit guten Gründen auf allen Feldern des öffentlichrechtlich organisierten Familienlasten- und -leistungsausgleichs noch quantitativ mehr und qualitativ Besseres gefordert werden; wegen der überreichen Fülle an einschlägigem Material und familienpolitischen Initiativen wird aber hierauf an dieser Stelle nicht weiter eingegangen. Stattdessen werden neben einigen Einrichtungen des Sozialrechts vorwiegend neuere Entwicklungen von internalisierenden Bedarfsdeckungsinstrumenten ${ }^{9}$ in den Blick genommen, also Änderungen des privaten Familien- und Erbrechts unter der engführenden Perspektive, ob und inwieweit durch sie privat familiäre Ressourcen, materieller wie immaterieller Art, Entlastungswirkungen zugunsten öffentlicher Kassen erzeugen. Es geht mit anderen Worten um Eigenverantwortung in Gestalt von „Familiensolidarität“, mithin auch um Ausläufer und Randerscheinungen des aktuellen Konzepts vom ,aktivierenden Sozialstaat“. ${ }^{10}$

Der gleichermaßen in der sozialpolitischen Rhetorik wie im rechtswissenschaftlichen Diskurs gern gebrauchte Begriff der Familiensolidarität vereinigt in sich die facettenreichen und geradezu gegensätzlichen Sinngehalte des normativ verbindlichen wie des rein faktischen, des rechtlich wie des sittlich gebotenen und eines lediglich erwünschten Verhaltens in familiären Zusammenhängen. ${ }^{11}$ Solch changierendem Verständnis gemäß kann „Familiensolidarität“, ungeachtet der subjektiven Einstellung der Beteiligten zueinander, äußerstenfalls imperativ und unmittelbar angeordnet werden, wie es hauptsächlich durch die Festsetzung von Unterhaltspflichten und von Grenzen der Testierfreiheit geschieht. Anknüpfend an tatsächlich gelebte oder auch nur vermutete Familiensolidarität können Sozialleistungen begrenzt werden oder umgekehrt kann familiensolidarisches Verhalten mittelbar durch dispositives Recht und inzentiv lenkende Angebote gefördert werden. Beispiele hierfür bilden etwa Sozialleistungen wie Pflegegeld oder auch Gestaltungsoptionen in den Eltern-Kind-Beziehungen und im Erbrecht. Schließlich können sich, gleichsam unter der Hand oder wenigstens nicht offen ins sozial- und familienpolitische Kalkül gezogen, günstige Nebenwirkungen einstellen, wo anderweitige hochrangige Zielvorstellungen, wie vornehmlich Kindeswohl, Geschlechtergleichstellung, Teilhabe- und Verteilungsgerechtigkeit in Bezug auf privates Einkommen und Vermögen, die Rechtspolitik antreiben.

Dem älteren Sozialrechtsdenken ist vor allem die Aufmerksamkeit für die gegenläufige Entwicklungstendenz vertraut, wie sie mit der Bismarckschen Arbeiterversicherung

9 Grundlegend zur Regelungs-Dichotomie ,internalisierend/externalisierend“ Zacher, Hans F., Zur Anatomie des Sozialrechts, SGb 1982, S. 329.

10 Hierzu Kingreen (Fußn. 1), JZ 2004, S.946 f.; ders. u. a., Aktivierung und Prävention - Chancen für Effizienzsteigerung in den Sozialleistungsbereichen, SDSRV 52 (2004); Eichenhofer, Eberhard, Sozialreformen zwischen Vision und Wirklichkeit, NZS 2007, S. 57, 60; soziologisch grundlegend Lessenich, Stefan, Die Neuerfindung des Sozialen, Bielefeld 2008.

11 Zur Vagheit und Offenheit der Begriffe „Solidarität“ und „soziale Gerechtigkeit“, die mehr einen Auftrag zur Problemlösung als diese selbst skizzieren Zacher, Hans F., Kinder und Zukunft - Einige Anmerkungen zur intergenerationellen Solidarität, in: Lorenz (Hrsg.), Festschrift für Andreas Heldrich, München 2005, S. 1211. 
begann: die Verdrängung des Zivilrechts durch das Sozialrecht ${ }^{12}$ im Sinne des Rückgangs von dessen tatsächlichem Anwendungsbereich. Das Hauptbeispiel der Unterhaltsverdrängung ist die Kollektivierung der Alters- und Hinterbliebenenversorgung. Ähnlich wirken alle sozialversicherungsrechtlichen Lohnersatzleistungen, aber auch die krankenversicherungsrechtliche Familienversicherung, die Ausbildungsförderung, die Grundsicherung für Alte und Erwerbsgeminderte. ${ }^{13}$ Die letzte sozialpolitische Großaktion in dieser Wirkrichtung stellt die Einführung der Pflegeversicherung dar - zur Kennzeichnung ihrer Zivilrechtsrelevanz sei an dieser Stelle nur das kritische Schlagwort „Erbenschutzversicherung" 14 genannt.

\section{Monetäre Anerkennung für private Langzeitpflege und Kleinkind- betreuung}

1. Diesem jüngsten Zweig der deutschen Sozialversicherung wurde bereits von Anfang an eine ausgeprägte Komponente der Anerkennung und Förderung von privater Pflegebereitschaft eingefügt: hauptsächlich durch die Gewährung von bescheidenem Pflegegeld für selbst beschaffte Pflegehilfen (§ 37 SGB XI) und einem Beitrag zur Alterssicherung der Pflegepersonen (§ 44 SGB XI). Mit dem am 1. Juli 2008 in Kraft getretenen Pflege-Weiterentwicklungsgesetz ${ }^{15}$ wird zur künftigen Erhaltung und möglichst noch größeren Verbreitung der privaten Angehörigenpflege wegen deren erheblicher Entlastungswirkungen für die Pflegeversicherung und sonstige öffentliche Kassen die Förderung weiter ausgebaut, indem u. a. die Pflegegelder stufenweise erhöht und schließlich dynamisiert werden ( $\S 30,37$ Abs. 1 SGB XI), ferner die Leistungen für demente Versicherte in häuslicher Pflege verbessert ( $§ 45$ a ff. SGB XI ) und sonstige Unterstützungsvorkehrungen, wie insbesondere die Einrichtung von Pflegestützpunkten (§ 92 c SGB XI), getroffen werden. ${ }^{16}$

12 Dazu Gitter, Wolfgang/Hahn-Kemmler, Jutta, Die Verdrängung des Zivilrechts durch das Sozialrecht - dargestellt am Unterhaltsrecht, SGb 1979, S. 195; Fuchs, Maximilian, Zivilrecht und Sozialrecht, 1992, S. 304 ff.; Zacher, in: Lorenz (Hrsg.), Festschrift für Andreas Heldrich (Fußn. 11), S. 1212; zum Gesamtkomplex wechselseitiger Beeinflussung umfassend monografisch Ruland, Franz, Familiärer Unterhalt und Leistungen der sozialen Sicherheit, München 1973.

13 Das prominenteste, weil gesetzlich dekretierte Zivilrechts-Verdrängungsbeispiel ist indessen die Ablösung der Haftung von Unternehmern und Arbeitskollegen durch die gesetzliche Unfallversicherung ( $§ 104 \mathrm{ff}$. SGB VII).

14 Grünenfraktion stellt Pflegeversicherung in Frage, Handelsblatt v. 6.8.2003; Berliner Zeitung, Textarchiv v. 7.8.2003; Baureithel, Ulrike, Der Pflegefall Pflegekasse, Das Parlament, Beil. „Aus Politik und Zeitgeschichte" v. 23.2. 2004.

15 Gesetz zur strukturellen Weiterentwicklung der Pflegeversicherung v. 28.5.2008, BGB1 I S. 874.

16 Von den Ausläufern dieser „Pflege-Privatisierungsinitiative“ ins private Erbrecht hinein wird am Schluss noch die Rede sein (VI.). 
2. Dasselbe inzentive Regelungsmodell liegt dem nach heftigem politischen Ringen nun ab dem Jahr 2013 gesetzlich in Aussicht gestellten „Betreuungsgeld“ in Höhe von 150 Euro (oder einem entsprechenden Wertgutschein) zugrunde, das für die häusliche Betreuung von Kleinkindern zwischen eins und drei Jahren anstatt der Inanspruchnahme einer Betreuungseinrichtung gewährt werden soll. ${ }^{17}$

3. Nicht diesem modernen Muster einer monetären Honorierung von sozialfiskalisch günstigen Verhaltensweisen im familiären Bereich folgen hingegen die älteren, undifferenzierten Leistungsausschlüsse für die Sozialversicherungsleistungen der häuslichen Krankenpflege und der Haushaltshilfe (insbes. §§ 37, 38 SGB V, § 32 SGB VII), soweit eine im Haushalt des kranken Versicherten lebende Person die erforderliche Krankenpflege und -versorgung bzw. die Haushaltsführung übernehmen kann. ${ }^{18}$ Diese heutzutage ausgesprochen harsch anmutende Indienstnahme von Familien- und Haushaltsangehörigen der Versicherten kann als verfassungsgemäß wohl allenfalls für die im Wege der Familienversicherung ( $§ 10$ SGB V) in den beitragslosen Versicherungsschutz einbezogenen Angehörigen der Kernfamilie aufrechterhalten werden. ${ }^{19}$ Noch restriktiver ist das Bundessozialgericht in neuerer Zeit vorgegangen: Fast unverhohlen rechtsfortbildend hat es dieser gesetzlichen Anforderung an die familiäre Solidarität praktisch vollends den Gebotscharakter genommen, indem es den Leistungsausschluss nach $§ 37$ Abs. 3 SGB V nurmehr für begründet hält, wenn - dokumentiert durch die tatsächliche Erbringung der Pflege! - die aktive und die passive Pflegewilligkeit von versicherter und haushaltsangehöriger Person vorliegen. ${ }^{20}$ De lege ferenda gibt das Gericht noch zu erwägen, ob nicht die Bereitschaft von Angehörigen zur Krankenpflege ebenfalls durch Pflegegeld gefördert werden sollte. ${ }^{21}$

17 Nur grundsätzliche Festlegung im Entwurf des neuen „Kinderförderungsgesetzes“ (KiFöG), BRDrs. 295/08 B a. E., Art. 1: $§ 16$ Abs. 4 SBG VIII-E.

$18 \S \S 37$ Abs. 3, 38 Abs. 3 SGB V; $\S 32$ Abs. 3 SGB VII; $\S 198$ RVO betr. Mutterschaftspflege. Zu weiteren entsprechenden Anwendungen kraft gesetzlicher Verweisung und zum Ganzen demnächst Frey, Michael, Häusliche Krankenpflege und Selbsthilfevorbehalt - Entwicklung, verfassungsrechtliche Fragen und rechtspolitische Folgerungen, (Diss.) Freiburg 2009.

19 So schon überzeugend Schulin, Bertram, in HS-KV, München 1993, § 6 Rdnr. 64.

20 BSGE 86, 101, 105, mit der Formulierung: ,,ist „hinter seinem Wortlaut zurückbleibend“ dahin auszulegen (...) Ein „Gebotsrest“ bleibt bei Pflegeverweigerung aus nicht nachvollziehbaren Gründen sowie bei kollusivem Zusammenwirken. “ Zur früheren Rspr., vor allem die Hilfepflicht des Ehegatten bei der Heimdialyse betreffend Poske, Dieter, Hauspflege, Baden-Baden 1990, S. 188 ff.

21 BSGE 86, 105; ausführlich für diese Gestaltung werbend Frey, Häusliche Krankenpflege und Selbsthilfevorbehalt (Fußn. 18), Kap. 4. 


\section{Ausbau der Familiensolidarität zugunsten von Kindern - Überforde- rungstendenz bei „Patchworkfamilien“}

1. Minderjährigen Kindern kommt - rechtlich wie tatsächlich und sozialethisch unangefochten - das Höchstmaß an familiärer Versorgung und Förderung zu. Der Problemfokus liegt auf der, auch zukunftsgerichteten, Existenzsicherung von Kindern, die in unvollständigen, zerrütteten oder sog. Patchworkfamilien leben. Das Monitum unseres großen Lehrmeisters Zacher bezüglich des Sozialleistungsspektrums ${ }^{22}$, nicht einseitig nur auf die Versorgung mit Geldmitteln zu achten, ist auch auf diesem Gebiet zu beherzigen. Neben der monetären Versorgung ist das Bemühen um Stärkung des persönlichen Beziehungs- und Fürsorgenetzes zugunsten von Minderjährigen in unvollständigen, zerbrochenen und (oft wechselnd) atypisch zusammengesetzten Familien zu würdigen.

2. Besonders wichtig erscheint insoweit das Aufbrechen des überkommenen QuasiSorgerechtsmonopols der Mütter, womit rechtlich die Türen für mannigfaltige Möglichkeiten zur Einbeziehung der Väter in die Sorge- und Erziehungsarbeit ohne (fortbestehende) Ehe der Eltern weit geöffnet worden sind. Die gemeinsame elterliche Sorge ist - normativ wie faktisch - nach Trennung und Scheidung der Eltern inzwischen die Regel ( $\S 1626,1671$ BGB). Nicht miteinander verheiratete Eltern können die gemeinsame Sorge wählen (§ 1626a BGB), und ein „lediger“ Vater darf - nach jüngstem Spruch des Europäischen Gerichtshofs für Menschenrechte - nicht mehr allein aufgrund des entgegenstehenden Willens der Mutter von der elterlichen Sorge ausgeschlossen bleiben. ${ }^{23}$ Auch Stiefelternteilen sind gewisse sorgerechtliche Befugnisse eingeräumt (§ 1687b BGB).

Ein Umgangsrecht hat das Kind grundsätzlich auch mit dem nichtsorgeberechtigten Elternteil, und jeder Elternteil ist zum Umgang mit dem Kind verpflichtet und berechtigt (§ 1684 BGB). Das neue familiengerichtliche Verfahren ${ }^{24}$ soll sowohl die Chancen einer zügigen Klärung des Umgangsrechts wie auch seiner störungsfreien Durchsetzung 25 erheblich verbessern, um die Eltern-Kind-Beziehung möglichst intakt zu halten. Die sozialfiskalisch nüchterne Kostenbetrachtung hat freilich den zusätzlichen Aufwand in Rechnung zu stellen, der bei Leistungsempfängern nach SGB II und SGB XII wegen der nicht selten größeren Distanzen der elterlichen Wohnorte für die Ausübung des

22 Generell die „monetäre Schlagseite“ von Sozialpolitik und -recht sowie die Gründe dafür charakterisierend Zacher (Fußn. 9), SGb 1982, S. 335.

23 EGMR vom 3.12.2009; anders BVerfG FamRZ 2003, S. 285. Eingehend zur Reformdiskussion Coester, Michael, Nichteheliche Elternschaft und Sorgerecht, FamRZ 2007, S. 1137.

24 Gesetz zur Reform des Verfahrens in Familiensachen und in den Angelegenheiten der Freiwilligen Gerichtsbarkeit (FGG-Reformgesetz - FGG-RG) v. 27.6.2008 (BT-Drs.16/9733); Gesetzentwurf der Bundesregierung, BT-Drs. 16/6308 v. 7.9.2007.

25 Stichworte: Verfahrensbeistand, Ordnungsmittel statt Zwangsmittel, Umgangspfleger. 
Umgangsrechts zwangsläufig entsteht. ${ }^{26}$ Die Zuerkennung von Umgangsrechten an Dritte wie Großeltern, Geschwister und sogar nichtverwandte Personen wie ehemalige Pflege- oder Stiefeltern ( $\S 1685$ BGB) kann ebenfalls mithelfen, einer emotionalen Entwurzelung und Verarmung der Kinder infolge der Instabilität moderner Elternbeziehungen vorzubeugen und entgegenzuwirken. Als Kehrseite ist indes auch die Gefahr zu sehen, dass Kinder wegen der Vielfalt und Varianz (sozial-)familiärer Beziehungen ${ }^{27}$ einem erhöhten Streitpotential ausgesetzt werden können, was sie insgesamt nicht mehr zur Ruhe kommen lässt. ${ }^{28}$

3. Die tatsächlichen Chancen insbesondere von Kleinkindern bis zu drei Jahren auf elterliche, typischerweise mütterliche Betreuung haben sich durch den seit 1998 normierten Anspruch auf sog. Betreuungsunterhalt des betreuenden Elternteils gegen den nicht betreuenden Elternteil ( $\$ 16151$ BGB), ${ }^{29}$ meist den Vater eines nichtehelichen Kindes, nicht sonderlich erhöht. ${ }^{30}$ Seine Bedeutung ist durch die vom BVerfG ${ }^{31}$ geforderte und inzwischen gesetzlich verankerte Gleichstellung der mit dem Vater des Kindes verheirateten und der nichtverheirateten Mütter aber zumindest angestiegen.

4. Kernbereich der zwingend auferlegten Familiensolidarität ist das Unterhaltsrecht. Seit Anfang des Jahres 2008 begründet das novellierte Unterhaltsrecht ${ }^{32}$ einen generellen Unterhaltsvorrang von minderjährigen Kindern, gleichgültig ob sie aus ehelicher oder nichtehelicher Verbindung stammen (§ 1609 BGB). Selbst Ehegatten stehen in den weitverbreiteten „Mangellagen“ hinter Kindern des Unterhaltsschuldners zurück. Dies entspricht dem strikt verstandenen Kindergleichstellungsgebot des Art. 6 Abs. 5 GG

26 Auf die Schwierigkeiten einer rechtlich tragfähigen Begründung und Durchführung, insbesondere mithilfe der Auffanglösung über $§ 73$ SGB XII auch für „Hartz IV-Empfänger“ sei hier nur pauschal hingewiesen; vgl. BSG 27.1.2009, B 14 - 11b AS 9/07 Rz. 45; Münder, Johannes, in: ders. (Hrsg.), LPK-SGB II, 3. Aufl. Baden-Baden 2009, § 23 Rdnr. 5, 6, 9.

27 Plastisch die Formulierungen von Coester-Waltjen, Dagmar, Kinderarm, aber elternreich, in: Hofer (Hrsg.), Festschrift für Dieter Schwab zum 70. Geburtstag am 15. August 2005, Bielefeld 2005, S. 761 („Das Kaleidoskop sorgerechtlicher Befugnisse“ a. a. O.).

28 So Hohmann-Dennhardt, Christine, Familienrechtliche Antworten auf veränderte Familienwelten, Forum Familienrecht (FF) 2007, S. 174, 184; gegenüber der starken Betonung von Rechtspositionen bedenklich auch Di Fabio, Udo, Der Schutz von Ehe und Familie: Verfassungsentscheidung für die vitale Gesellschaft, NJW 2003, S. 993, 995 f.; Schwab, Dieter, Familienrecht, 17. Aufl. München 2009, Rdnr. 763.

29 Dazu näher (Rechtslage vor dem 1.1.2008) und mit der Kritik, es handle sich um ein Konzept, Mütter durch Unterhaltsversprechungen vom Arbeitsmarkt fernzuhalten, Springer, Astrid, Unterhalt für die Mutter eines nichtehelichen Kindes, in: Berghahn (Hrsg.), Unterhalt und Existenzsicherung, Baden-Baden 2007, S. 81.

30 Nach Bundesfamilienministerium, Monitor Familienforschung, Ausgabe 15, November 2008, S. 12, lebten im Jahr 2007 nur weniger als $5 \%$ der alleinerziehenden Frauen von familiärem Unterhalt.

31 BVerfG, NJW 2007, S. 1735 (mit Anmerkung Caspary).

32 Gesetz zur Änderung des Unterhaltsrechts v. 21.12.2007, BGB1 I S. 3189. Dazu Born, Winfried, Das neue Unterhaltsrecht, NJW 2008, S. 1; Derleder, Peter, Die Unterhaltsreform als Neustrukturierung von Ehe und Familie, in: Berghahn (Hrsg.), Unterhalt und Existenzsicherung, Baden-Baden, 2007, S. 153. 
und mag häufig besonders dem Wohl der nichtehelichen oder zweit- und drittehelichen Kinder dienen. ${ }^{33}$ Es mindert aber auch die öffentliche Auffangverantwortung für nicht oder nur defizitär versorgte Kinder, welche durch das Unterhaltsvorschussgesetz sowie SGB II und SGB XII wahrgenommen wird. Denn im Gegensatz zu Erwachsenen können Kinder nicht auf Eigenverantwortung und Erwerbsobliegenheiten verwiesen werden; sie fallen dem Staat daher - zumindest statistisch - unabwendbarer zur Last. ${ }^{34}$

Diese staatliche Last in Stiefeltern- und sog. „Patchworkfamilien“ bei der Prüfung der Sozialgeldberechtigung von Kindern durch die unabwendbare Anrechnung von Einkommen auch des Stiefelternteils oder sogar des nichtehelichen Partners ihres leiblichen Elternteils zu erleichtern, wie es durch die Änderung von $\S 9$ Abs. 2 S.2 SGB II bestimmt worden ist ${ }^{35}$, stößt auf verbreitete und vollauf berechtigte Kritik ${ }^{36}$. Wir haben hier wohl den derzeit erstaunlichsten Zugriff auf private Ressourcen zur Schonung öffentlicher Mittel vor uns, da ja privatrechtliche Unterhaltspflichten gegenüber dem Partnerkind kraft Gesetzes gerade nicht bestehen ${ }^{37}$ und auch von einer selbstverständlichen sozialen Unterhaltspraxis insoweit nicht die Rede sein kann. ${ }^{38}$ Daher verstößt mehr als eine widerlegliche Vermutung der tatsächlichen Unterhaltsgewährung an das Stief- oder Partnerkind sowohl gegen die staatliche Pflicht zur Gewährleistung des Existenzminimums des Kindes (Art.1 Abs. 1 GG i.V.m. Sozialstaatsgebot) wie auch gegen die Handlungsfreiheit des leiblichen Elternteils und des Partners (Art. 2 Abs. 1GG).

Die geplante Reform des EU-Gemeinschaftsrechts im Bereich der Unterhaltspflichten wird vor allem für die unkompliziertere Durchsetzung von Kindesunterhaltsansprüchen von Nutzen sein. 39

33 Wo ein Kind (etwa aus erster Ehe) und sein betreuender Elternteil in einem Haushalt leben, stehen ihnen im Mangelfall unterm Strich nicht mehr Mittel zur Verfügung.

34 Vgl. auch Lenze, Anne, Das neue Unterhaltsrecht aus sozialrechtlicher Perspektive, FamRZ 2009, S. 1724, m. Hinw. auf BT-Drs. 16/ 1830, S. 1, 16. Auch zu Rücksichten auf öffentliche Lasten im Zuge der historischen BGB-Gesetzgebung Lipp, Volker, Funktion und Bedeutung des Unterhaltsrechts, in: Behrends/Schumann (Hrsg.), Gesetzgebung, Menschenbild und Sozialmodell im Familien- und Sozialrecht, Berlin/New York 2008, S. 133.

35 Zur Vorgeschichte eingehend Hänlein, Andreas, in: Gagel, SGB II-SGB III, 34. EL, München 2009, SGB II § 9 Rdnr. $48 \mathrm{f}$.

36 Frühzeitig für grundgesetzwidrig erachtet von Wenner, Ulrich, Verfassungsrechtlich problematische Regelungen für eheähnliche Gemeinschaften und Stiefeltern, Soziale Sicherheit 2006, S.146; SG Berlin, B. v. 8.1.2007, info also 2007, Heft 3, m. zust. Anm. Spindler, Helga. Vgl. gesetzeskrit. auch Rosenow, Roland, Bedürftigkeitsfiktion und Verteilung von Einkommen innerhalb der Bedarfsgemeinschaft im SGB II, SGb 2008, S. 282; Lenze, Anne, Das neue Unterhaltsrecht aus sozialrechtlicher Perspektive, FamRZ 2009, S. 1724, 1726.

37 Was merkwürdigerweise das BSG nicht klar zu erkennen scheint, BSG 13.11.2008, B 14 AS 2/08 R, NZS 2009, S. 634 = FamRZ 2009, S. 1057; rechtsdogmatische Kritik von Münder, Johannes/Geiger,Udo, Die generelle Einstandspflicht für Partnerkinder in der Bedarfsgemeinschaft nach $\S 9$ Abs.2 Satz 2 SGB II, NZS 2009, S. 593, 596.

38 Allerdings begründet beim Arbeitslosengeld I auch ein Stief- oder Partnerkind im Haushalt des Arbeitslosen den erhöhten Leistungssatz von $67 \%$ (§ 129 Nr. 1 SGB III).

39 Mitteilung 2005 / NaN EG v. 15.12. 2005. 
5. Nicht nur der Kuriosität halber sei noch angefügt, dass eine infolge der modernen Fortpflanzungstechniken nicht mehr auszuschließende Unsicherheit über die mütterliche Abstammung eines Kindes (und damit die Gefahr einer Kindeswohlgefährdung) vom Gesetz als schlechthin irrelevant beiseite geschoben wird. Apodiktisch und ausnahmslos bestimmt $\S 1591$ BGB: „Mutter eines Kindes ist die Frau, die es geboren hat.“ Allein sie trifft mit der Geburt die volle mütterliche Unterhalts- und Sorgeverantwortung, der sie sich allenfalls durch Freigabe des Kindes zur Adoption entledigen kann. Dem Kind freilich darf die Kenntniserlangung über seine genetische Abstammung als solche - gestützt auf Art. 1, 2 GG und dem einfachen Gesetzgeber insoweit unverfügbar - nicht endgültig verwehrt werden. ${ }^{40}$ Aufgrund öffentlicher Interessen bleibt die genetische Abstammung auch im Strafrecht ( $§ 173$ StGB) und im Eheschließungsrecht (§ 1307 BGB) maßgeblich. 41

\section{Unterhaltsbeziehungen zwischen erwachsenen Kindern und Eltern}

1. Zum Deszendentenunterhalt bewende es hier mangels aktueller Neuerungen oder Reformvorhaben bei knappen Hinweisen. Die deutschen Unterhaltspflichten der Eltern gegenüber ihren volljährigen Kindern (und grundsätzlich auch gegenüber ferneren $\mathrm{Ab}$ kömmlingen) gehören zu den zeitlich ausgedehntesten vergleichbarer Länder. ${ }^{42}$ Rein normativ und grundsätzlich bestehen sie lebenslänglich (§ 1601 BGB), nach Erreichen der Volljährigkeit liegt der Schwerpunkt beim Ausbildungsunterhalt. ${ }^{43}$ Trotz der sozialrechtlichen Ausbildungsförderung (BAföG) haben zu großen Anteilen die Eltern die Hochschulausbildung ihrer Kinder zu finanzieren (und nicht ganz selten sogar eine zweite Ausbildung), die bis weit in das dritte Lebensjahrzehnt hinein andauert. Gelangen erwachsene, unverheiratete Kinder nicht dauerhaft zu wirtschaftlicher Selbständigkeit, haben leistungsfähige Eltern weiterhin vorrangig in die Bresche zu springen. Die Gewährung bedürftigkeitsabhängiger Sozialleistungen aufgrund SGB II und SGB XII kann Regressansprüche gegen die Eltern nach sich ziehen. Recht großzügig befreit von

40 Für die väterliche Abstammung: BVerfG, NJW 1989, S. 891; BVerfG, NJW 1994, S. 2475. Bezüglich der mütterlichen Abstammung müsste daher wohl die Möglichkeit einer Feststellungsklage nach § 256 ZPO (ohne Statusfolgen) eröffnet sein (so BT-Drs. 13/4899, S. 83); abl. und zur Problematik Staudinger-Rauscher (2004), § 1591 Rdnr. 20 ff.; Gernhuber, Joachim/Coester-Waltjen, Dagmar, Familienrecht, 6. Aufl. München 2010, § 51 Rdnr. 7-9.

41 Palandt/Diederichsen, BGB, 68. Aufl. München 2009, § 1591 Rdnr. 2, 3.

42 Vgl. Schwab, Dieter/Henrich, Dieter (Hrsg.), Familiäre Solidarität - Die Begründung und die Grenzen der Unterhaltspflicht unter Verwandten im europäischen Vergleich, Bielefeld 1997; Brudermüller, Gerd, Solidarität und Subsidiarität im Verwandtenunterhalt - Überlegungen aus rechtsethischer Sicht, FamRZ 1996, S. 129; Frank, Rainer, Familiäre Solidarität im Unterhalts- und Erbrecht, FamRZ 2009, S. 649, 651.

43 Eingehend dazu und zur Kritik Schwab, Dieter, Das deutsche Recht, in: ders./Henrich (Hrsg.), Familiäre Solidarität (Fußn. 42), S. 39, 44 ff. 
solch weitreichender Unterhaltsverantwortung - bis auf geringe Pauschbeträge von derzeit 26 und 20 Euro monatlich - sind die Eltern von volljährigen behinderten oder pflegebedürftigen Kindern (§ 94 Abs. 2 SGB XII). ${ }^{44}$

2. Zum Aszendentenunterhalt ist herauszustellen, dass sich in neuerer Zeit eine beachtliche Auflockerung der rechtlich angeordneten Familiensolidarität im Zusammenspiel von Sozialrecht und Familienrecht ereignet hat. Im Ausgangspunkt hält bislang allerdings, das in der Generationenfolge prinzipiell nach oben und unten unbegrenzte Verwandtenunterhaltsrecht ( $\S \S 1601$ ff. BGB) allem gesellschaftlichen Wandel stand, wie er vornehmlich durch die Entwicklung zur Klein- und Kleinstfamilie sowie zur generellen „Individualisierung“ gekennzeichnet ist. Rechtspolitische Radikalforderungen nach gänzlicher Abschaffung des Elternunterhalts 45 haben auch gegenwärtig keine reale Aussicht durchzudringen. ${ }^{46}$ Die stattgefundene Ermäßigung beruht zum einen auf der expliziten Zurückführung der sozialhilferechtlichen Regressmöglichkeiten weit hinter die familienrechtlichen Unterhaltspflichten und zum anderen auf der flexiblen Tatbestandsfassung und rücksichtsvollen Konkretisierung der familienrechtlichen Anspruchsnorm mit dem zentralen Merkmal der Leistungsfähigkeit des (potentiellen) Unterhaltsschuldners (§ 1603 Abs. 1 BGB).

Familiäre Solidarität zwischen Verwandten zweiten Grades (Großeltern-Enkel) und höheren Grades wird sozialrechtlich überhaupt nicht mehr auferlegt; Sozialhilfeleistungen lösen in diesen Beziehungen keine Regressansprüche mehr aus (§ 94 Abs. 1 S. 3 SGB XII).

Für Menschen im regulären Rentenalter oder mit voller Erwerbsminderung ist der Unterhaltsregress gegen ihre grundsätzlich unterhaltspflichtigen Kinder durch die besondere Sozialhilfeart der „Grundsicherung im Alter und bei Erwerbsminderung“ $(\S \S 41 \text { ff. SGB XII })^{47}$ erheblich reduziert worden. ${ }^{48}$ Nimmt eine bedürftige Person diese Grundsicherung in Anspruch, so ist ein ihr unterhaltspflichtiges Kind einem sozialhilferechtlichen Rückgriffsanspruch erst ab einem Jahreseinkommen von 100.000 Euro ausgesetzt ( $\$ 43$ Abs. 2 S. 1 SGB XII $)^{49}$, wobei zugunsten des Kindes noch die gesetzliche Vermutung gilt, dass diese Einkommensgrenze nicht überschritten wird. Der Ver-

44 Dazu und zu vorausgehenden Differenzierungen Müller, Christian, Der Rückgriff gegen Angehörige von Sozialleistungsempfängern, 5. Aufl. Baden-Baden 2008, Teil B, Rdnr. 23 ff., 32.

45 So Beschlüsse des 59. DJT 1992 zu C. II.1. (angenommen mit 47:37:4); ähnlich Brudermüller, Gerd, Solidarität und Subsidiarität im Verwandtenunterhalt - Überlegungen aus rechtsethischer Sicht, FamRZ 1996, S. 129, 133 f.

46 Vgl. auch Martiny, Dieter, Verwandtenunterhalt für erwachsene Kinder und alte Eltern, in: Berghahn (Hrsg.), Unterhalt und Existenzsicherung (Fußn. 29), S. 54, 64.

47 Eingeführt ursprünglich durch das am 1.1.2003 in Kraft getretene „Gesetz über eine bedarfsorientierte Grundsicherung im Alter und bei Erwerbsunfähigkeit“(GSiG), BGB1 2001 I S. 1335.

48 Sozialpolitisch krit. zu diesem Ansatz von Koppenfels-Spies, Katharina, Abschied von Solidarität und Subsidiarität - Das neue Grundsicherungsgesetz, FPR 2003, S. 341.

49 Auch bei mehreren Kindern gilt diese Schongrenze für jedes einzelne Kind, Brühl/Schoch in LPKSGB XII, 7. Aufl. Baden-Baden 2005, § 43 Rdnr. 9; DV, NDV 2002, S. 341, 342. 
mögensstamm des Kindes bleibt ganz außer Betracht. Allerdings erstreckt sich die Grundsicherung, was gelegentlich verkannt wird, nur auf die Kosten des allgemeinen Lebensunterhalts (§ 42 SGB XII) und umfasst gerade nicht die drückendsten Belastungen im Bereich des Aszendentenunterhalts, die aufgrund hoher und tendenziell ansteigender Heimpflegekosten anfallen können. ${ }^{50}$ Doch wird den Kindern pflegebedürftiger Eltern eine spürbare Lastenbegrenzung nicht erst von der Sozialhilfeseite, sondern bereits von der Unterhaltsseite her zuteil, nämlich durch eine insgesamt moderate Zuerkennung elterlicher Unterhaltsansprüche; auf das feinmaschige richterrechtliche Regelwerk und die Auseinandersetzung damit im Schrifttum kann hier nur hingewiesen werden. ${ }^{51}$ Als Richtschnur gilt, dass der Unterhaltsverpflichtete eine spürbare und dauerhafte Senkung seines berufs- und einkommenstypischen Lebenshaltungsniveaus jedenfalls insoweit nicht hinzunehmen braucht, als er nicht einen nach den Verhältnissen unangemessenen Aufwand betreibt oder ein Leben im Luxus führt. 52

Wo immer der Sozialhilfe-Unterhaltsregress unterhalb der familienrechtlichen Einstandsschwellen bleibt, wird in sehr eigentümlicher Weise ein Stück ,privater Unterhaltsautonomie“" aus der Hand des Sozialrechtsgesetzgebers gewährt. Und mutmaßlich bewirken nicht zuletzt gerade solche Familienverhältnisse, in denen, weil wenig oder keine lebendige Solidarität (mehr) herrscht, harte Unterhaltskonflikte ausgetragen werden, im Ergebnis Entlastungen der Sozialhilfe.

\section{Ambivalente Tendenzen in Bezug auf nicht (mehr) verheiratete Partner}

1. Hält man im „Familien - Partnerschaftsrecht“ Ausschau nach Entwicklungen von sozialfiskalischer Schutztendenz oder zumindest Günstigkeit, zeigt sich hinsichtlich der i. w. S. vermögensrechtlichen Einrichtungen für geschiedene Eheleute ein janusköpfiges Bild: retrospektiv verstärkte Vorkehrungen um die „gerechte“ und wirksame Teilhabe beider am wirtschaftlichen Ertrag der zurückliegenden Ehezeit, prospektiv aber ein Schwinden von nachehelicher Solidarität bezüglich künftigen Unterhaltsbedarfs. Der generelle Vorrang der Unterhaltsansprüche von Kindern wurde bereits erwähnt (s. o. III). In den Fällen der Konkurrenz von sich jeweils auf die Betreuung eines Kindes gründenden Partnerunterhaltsansprüchen ist es typischerweise die geschiedene Frau, die zeitlich als erste aus der Unterhaltsberechtigung wieder heraus fällt und auf die Wahrnehmung von Eigenverantwortung qua Erwerbstätigkeit verwiesen wird.

50 Vgl. allgemein Becker, Ulrich, Die alternde Gesellschaft - Recht im Wandel, JZ 2004, S. 929, $932 \mathrm{ff}$.

51 Dazu mit zahlreichen Nachweisen Martiny (Fußn. 46), in: Berghahn (Hrsg.), Unterhalt und Existenzsicherung, S. $63 \mathrm{ff}$.

52 BGH v. 23.10.2002, NJW 2003, S. 128, 130. 
2. Privatautonome Gestaltungen in Bezug auf nachehelichen Unterhalt sowie von Zugewinn- und Versorgungsausgleich zum Nachteil des wirtschaftlich schwächeren Partners (und damit oft auch der Kinder) werden seit längerem durch legislative wie judikative Schranken hintanzuhalten versucht; die im Jahr 2009 erfolgten Gesetzesänderungen setzen diese Linie fort.

So waren Vereinbarungen über den Versorgungsausgleich im Zusammenhang mit der Scheidung vom gesetzlichen Geltungsbeginn an nicht nur notariell zu beurkunden, sondern vom Familiengericht auch daraufhin zu überprüfen, ob sie unter Einbeziehung der Unterhaltsregelung und der Vermögensauseinandersetzung nicht offensichtlich das Sicherungsziel des Versorgungsausgleichs verfehlten (§ 1587o BGB a.F.). Der grundsätzlichen Freiheit zum ehevertraglichen Ausschluss des Versorgungsausgleichs (mindestens ein Jahr vor Scheidungsantragsstellung) sowie des Zugewinnausgleichs und nachehelicher Unterhaltsansprüche ( $\S 1408,1585 \mathrm{c}$ BGB) wurden durch die zwischenzeitlich auch verfassungsrechtlich sanktionierten Instrumente der Inhaltskontrolle und der Ausübungskontrolle Grenzen gezogen. ${ }^{53}$

Beruht diese Rechtsprechung insbesondere zum Verzicht auf nachehelichen Unterhalt wesentlich auch auf dem Schutz von Kindesinteressen, so fällte die Zivilrechtsprechung schon vor längerer Zeit das Verdikt der Nichtigkeit wegen Sittenwidrigkeit auch über solche Unterhaltsverzichtsvereinbarungen, die von den geschiedenen oder scheidungswilligen Eheleuten objektiv zum Nachteil der Sozialhilfe geschlossen werden. Dabei kommt es nicht auf eine Schädigungsabsicht an, sondern es genügt, dass sich die Parteien der Erkenntnis der drohenden Sozialhilfebedürftigkeit grob fahrlässig verschlossen haben. ${ }^{54}$

3. In das Tableau sozialstaatsentlastender „Familiensolidarität kraft Gesetzes“ fügen sich die jüngsten legislativen Ansätze zur Perfektionierung und Effektivierung der vermögensmäßigen Hauptausgleichsinstrumente bei der Scheidung, Zugewinnausgleich ${ }^{55}$ und Versorgungsausgleich ${ }^{56}$, und die damit zu erzielende Stärkung der wirtschaftlichen Selbständigkeit auch des häufig, wenngleich nicht notwendig ehebedingt schwächeren

53 Grundlegend BVerfG U. v. 6.2.2001, E 103, 8, NJW 2001, S. 957; BVerfG B. v. 29.3.2001, NJW 2001, S. 2248; danach eher ,pro Vertragsfreiheit“ argumentierend BGH v. 11.2.2004, Z 158, 81. Ein jüngst (9.7.2008) vom BGH entschiedener krasser Fall der Benachteiligung der schwangeren Ehefrau in FamRZ 2008, S. 2011.

54 BGH v. 24.4.1985, Z 86, 82,88; BGH v. 9.7.1992, FamRZ 1992, S. 1403; BGH v. 25.10.2006, NJW 2007, 904; MünchKomm-Maurer, 4. Aufl. München 2000, § 1585c BGB, Rdnr. 42; eher zurückhaltend Fuchs (Fußn. 12), S. 312 ff.; eingehend zu Entwicklung und dogmatischer Begründung Deinert, Olaf, Die Bedeutung des Sozialrechts für das Privatrecht am Beispiel des Verzichts auf nachehelichen Unterhalt, ZFSH/SGB 2008, S. 515.

55 BGB1 2009 I, S. 1696; zuvor Entwurf eines Gesetzes zur Änderung des Zugewinnausgleichs- und Vormundschaftsrechts v. 5.11.2008, BT-Drs. 16/10798; zum Überblick Büte, Dieter, Die Reform des Zugewinnausgleichsrechts, FamRZ 2009, S. 2776.

56 BGBl 2009 I, S. 700 mit nun eigenständigem Versorgungsausgleichsgesetz (VersAusglG); zuvor Entwurf eines Gesetzes zur Strukturreform des Versorgungsausgleichs (VAStrRefG) v. 20.8.2008, BT-Drs. 16/10144 und BT-Drs. 16/11903. 
Partners. Bei beiden Instrumenten ging es im Kern nicht um eine wertungsmäßig tiefgreifende Reform, sondern eher um die Nachjustierung von grundsätzlich als bewährt erachteten Rechtseinrichtungen im Sinne möglichst vollwertiger und praktikabler Verwirklichung des mittlerweile im herrschenden Sozialethos verankerten Prinzips der gleichen Teilhabe beider Ehegatten an dem in der Ehe erwirtschafteten Privat- wie Altersvorsorgevermögen.

$\mathrm{Zu}$ diesem Zweck werden nun im Zugewinnausgleich insbesondere auch bei Beginn des Güterstandes, i.d.R. also bei der Eheschließung vorhandene Schulden berücksichtigt (§ 1374 Abs. 3 BGB) sowie Vorkehrungen zum Schutz vor manipulativen Vermögensminderungen vor allem in zeitlicher Nähe zur Scheidung getroffen $(\S \S 1379,1390$ BGB); krisenbedingte Wertminderungen kurz vor Vollzug des Ausgleichs werden hingegen nicht berücksichtigt ${ }^{57}$.

Beim neugeordneten Versorgungsausgleich wird die für das Rentenalter angestrebte effektive Ergebnis-Teilhabe beider Ehegatten am ehezeitlich erworbenen Vorsorgevermögen im Wesentlichen durch die Umstrukturierung auf die reale systeminterne Teilung grundsätzlich sämtlicher ausgleichspflichtigen Versorgungsanrechte bewerkstelligt. ${ }^{58}$. Ferner wurde der Schutz des schwächeren Partners vor schädlichen Vereinbarungen, besonders dem Ausschluss des Versorgungsausgleichs, tendenziell wohl verfestigt, indem die mithilfe von $\S 138$ und $\S 242$ BGB judikativ entwickelten Instrumente einer Inhalts- und Ausübungskontrolle ${ }^{59}$ nun legislativ niedergelegt sind ( $\$ 8$ VersAusglG), freilich ohne jede maßstäbliche Konkretisierung im Gesetz. ${ }^{60}$

4. Die Beendigung nichtehelicher Partnerschaften durch Trennung oder Tod löst derzeit kraft Gesetzes noch keinerlei versorgungs-, güter- oder erbrechtliche Teilhabe am Partnervorsorge- und privatvermögen aus. Schon seit längerem wird von der Rechtsprechung immerhin ausnahmsweise bei Schaffung größerer und dauerhafter Vermögenswerte, wie etwa der Errichtung eines Familienheimes oder der Mithilfe beim Aufbau eines Unternehmens, ein gesellschaftsrechtlicher Ausgleich und neuerlich ein Anpassungsanspruch wegen Wegfalls der Geschäftsgrundlage bei „gemeinschaftsbezogenen Zuwendungen“ zugebilligt. ${ }^{61}$ Angesichts der Zunahme nichtehelicher Lebensge-

57 Krit. Schwab, Dieter, Neues im Familienrecht - Ein Zwischenbericht, FamRZ 2009, 1, 4.

58 Eingehend und krit. zu allen Reformaspekten Ruland, Franz, Die vorgesehene Strukturreform des Versorgungsausgleichs, NZS 2008, S. 225-237; ders., Der neue Versorgungsausgleich - eine kritische Analyse, NJW 2009, S. 2781.

59 Vgl. modellhaft noch BGH v. 18.3.2009, FamRZ 2009, S. 1041.

60 Zum derzeitigen Stand der richterlichen Vertragskontrolle Schwab, Familienrecht (Fußn. 28), Rdnr. 224 ff.; Gernhuber/Coester-Waltjen, Familienrecht (Fußn. 40), § 28 Rdnr. 18 ff.

61 BGH, FamRZ 1982, S. 1065; BGH, FamRZ 1985, S. 1232. - BGH 9.7.2008 Z 177, 193 Tz. 40 ff; für einen eheentsprechenden Ausgleich von „unbenannten Zuwendungen“ u. a. schon Frank, Rainer, Vermögensrechtliche Ansprüche beim Scheitern nichtehelicher Lebensgemeinschaften, in: Dieckmann u. a. (Hrsg.), Festschrift für Wolfram Müller-Freienfels, Baden-Baden 1986, S. 131. Überblick der filigranen Dogmatik möglicher Anspruchsgrundlagen bei Schwab, Familienrecht (Fußn. 28), Rdnr. 953 ff.; Gernhuber/Coester-Waltjen, Familienrecht (Fußn. 40), § 44 Rdnr. 20 ff. 
meinschaften, vornehmlich auch solcher mit Kindern, fehlt es nicht an Stimmen, die auf diesem Sektor weitergehende Fortentwicklungen nach dem Vorbild mancher ausländischer Regelungen rechtspolitisch anmahnen. ${ }^{62}$

5. Die unterhaltsrechtlich auffälligste Neuerung der jüngeren Zeit bildet der erwähnte Betreuungsunterhalt, der insbesondere das Armutsrisiko alleinerziehender Mütter mindert (§ 16151 BGB). Auch hierzu wurden zuletzt auf dem Deutschen Juristentag 2008 noch rechtspolitische Forderungen nach Verallgemeinerung und Verfestigung erhoben. ${ }^{63}$ Bei einer Konkurrenz von sich jeweils auf die Betreuung eines Kindes gründenden Partnerunterhaltsansprüchen ( $§ 1570,16151$ BGB) büßt die geschiedene Frau oder frühere Partnerin typischerweise zeitlich als erste ihre Unterhaltsberechtigung wieder ein, was jedoch die erwünschte Zusatzwirkung haben soll, dass die Betreuung von Kindern die Mütter nicht allzu lange vom Erwerbsleben fernhält. Mit dieser Neuverteilung der (weit überwiegend ja eher unzulänglichen) Ernährereinkommen birgt das reformierte Unterhaltsrecht zumindest auch sozialfiskalische und ökonomische Vorteile - vielleicht auch etwas mehr Chancen auf Nachwuchs, weil geschiedenen Männern familiäre „Altlasten“ erleichtert werden. ${ }^{64}$

6. In unserer Zusammenschau von existenzsichernden Einrichtungen des Familienund des Sozialrechts ist auch ein kurzer Blick auf den Unterhaltsersatz durch Hinterbliebenenrenten der gehobenen sozialen Sicherungssysteme, Sozialversicherung und soziale Entschädigung, zu werfen.

Ausschließlich Ehegatten und eingetragene Lebenspartner, nicht aber Partner sonstiger Lebensgemeinschaften, haben nach dem Tod des einen Anspruch auf Hinterbliebenenleistungen ( $\S \S 46$ SGB VI, 38 ff. BVG). Im Recht der gesetzlichen Unfallversicherung und der sozialen Entschädigung erstreckt sich der Schutz grundsätzlich auch noch auf unterhaltsberechtigte geschiedene Ehegatten ( $\S 66$ SGB VII, 42 BVG). In der gesetzlichen Rentenversicherung ist für geschiedene, kindererziehende Ehegatten immerhin die Sonderform der Erziehungsrente (§ 47 SGB VI) aus eigenen Rentenanwartschaften vorgesehen. Keinerlei Pendant dazu existiert jedoch für kinderbetreuende Elternteile, die mit dem anderen Elternteil nicht verheiratet waren, obwohl gemäß dem strikt verstandenen Kindergleichstellungsgebot des Art. 6 Abs. 5 GG die relativ neue bürger-

62 Dethloff, Nina, Familienarbeit im Wandel, in: Hofer/Klippel/Walter (Hrsg.), Perspektiven des Familienrechts, Festschrift für Dieter Schwab zum 70. Geburtstag am 15.8.2005, S. 349, 354 ff. (m. Hinw. auf die Erleichterung öffentl. Lasten); dies., Unterhalt, Zugewinn, Versorgungsausgleich - Sind unsere familienrechtlichen Ausgleichssysteme noch zeitgemäß?, Gutachten A 67. DJT 2008, A 35 ff. (zu ausländischen Regelungen), S. 148 ff., 159; dies., Familienrecht, 29. Aufl. München 2009, § 8 Rdnr. $43 \mathrm{ff}$.

63 Dethloff (Fußn. 62), 67. DJT, A 51 ff., 84, 154; Brudermüller, Gerd, Referat 67. DJT, Thesen I; zurückhaltend jedoch die Beschlüsse (A IV).

64 Vgl. auch Lenze (Fußn. 34), FamRZ 2009, S. 1726. Das höhere wirtschaftliche Risiko für die Frauen könnte jedoch auch zum gegenteiligen Effekt führen (so schon Schwab (Fußn. 1), FamRZ 2007, S. 4); ders., Mutter und Kinder bekommen am Ende weniger, Frankfurter Allgemeine Sonntagszeitung v. 25.3.2007, Nr. 12, S. 4. 
lichrechtliche Unterhaltsbeziehung, die ohne (vorausgehende) Ehe, nur an die Betreuung eines gemeinsamen Kindes anknüpft, inzwischen - auf verfassungsrechtlicher Grundlage! - fest etabliert ist. Diese gesetzliche Unterhaltsbeziehung im Falle des Todes des Unterhaltsschuldners auch durch eine Hinterbliebenenrente - nur für die gesetzliche Dauer der Betreuungszeit wohlgemerkt - zu flankieren, erscheint als Gebot wertungsmäßiger Konsequenz. ${ }^{65}$ Die Erziehungsrente gegenwärtiger Ausprägung erhärtet klar den Vorwurf, dass das Sozialversicherungsrecht noch immer zu einseitig und an dieser Stelle evident gleichheitswidrig den Schutz der Ehe betont - bei genauer Betrachtung bedürfte wohl die „Erziehungsrente“ generell einer Umstrukturierung. Angesichts des schroffen Gefälles in der Hinterbliebenensicherung erscheint aber auch die Möglichkeit nicht allzu fernliegend, dass bei weiterer Zunahme von nichtehelichen Partnerschaften mit Kindern der relativ hohe Hinterbliebenenschutz von jüngeren Ehegatten (selbst ohne Kinder) ${ }^{66}$ sich als sozialethisch nicht mehr gerechtfertigt darstellt, und das Gefälle künftig „,von oben her“ abgeflacht werden könnte.

\section{Normativ stabile Familiensolidarität im Erbrecht}

1. Erbrechtlicher Vermögensübergang stellt, wiewohl im Einzelfall meist „weder dem Grunde noch der Höhe nach" verlässlich und berechenbar zu erwarten, wenigstens nicht vor dem Erbfall, auf das gesellschaftliche Ganze gesehen doch auch einen erheblichen sozialstaatsentlastenden Privattransferstrom dar.

Das Erbrecht des BGB hat sich als die in dem Jahrhundert seiner Geltung stabilste Teilmaterie der Kodifikation erwiesen. Eine der wenigen Reformen auf diesem Gebiet bildet die etappenweise Angleichung der erbrechtlichen Stellung der nichtehelichen Kinder an die der ehelichen im Verhältnis auch zum Vater, die seit 1998 vollendet ist und somit manchem Menschen ein finanzielles Polster zukommen lässt, das ihm in prekärer Lage oder im Alter hilfreich sein kann und bedarfsabhängige Sozialleistungen entbehrlich macht. Sonstige Änderungen der gesetzlichen Erbfolge von Ehegatten, Abkömmlingen und etwa weitläufigen Verwandten ( §§ 1924 ff. BGB) stehen derzeit nicht auf der rechtspolitischen Agenda. 67

Über allen gesellschaftlichen Wandel hinweg versieht offenbar die Testierfreiheit das Gesamtsystem im Wesentlichen mit dem nötigen $\mathrm{Maß}$ an Elastizität. So sind es denn auch die Grenzen der Testierfreiheit, die mit dem Pflichtteilsrecht gezogen sind, um die

65 Inzwischen vertritt das Bay LSG (30.9.2009 L 1 R 204/09) in einem überaus eingehend begründeten Vorlagebeschluss zum BVerfG i.W. dieselbe Meinung.

66 Vgl. näher Köbl, Ursula, Familienleistungen in der Alterssicherung, DRV 2002, S. 686-696.

67 Zur Begrenzung der Verwandtenerbfolge in Verh. 49. DJT 1972 Coing, Helmut, I A 35 ff., und Dieckmann, Albrecht, II K $11 \mathrm{f}$. 
schon seit alters eine Gestaltungs- und Reformdiskussion geführt wird 68 - allerdings bezeichnenderweise ohne nennenswerten Erfolg in der Gesetzgebung. Denn auch beim Pflichtteilsrecht handelt es sich um ein Instrument der Vermögensverteilung unter Privaten, welches aufs große Ganze gesehen geeignet ist, Sozialtransfers zu erübrigen oder zu reduzieren.

Auch nach den Anfang des Jahres 2010 inkraftgetretenen Erbrechtsänderungen 69 bleibt das Pflichtteilsrecht in seiner hergebrachten Prägung erhalten. ${ }^{70}$ Umfangmäßig sollte es eine gewisse Schmälerung durch die Möglichkeiten zur nachträglichen Anordnung der Anrechnung und der Ausgleichung von Vorempfängen erfahren ( $\S 2315$ 2316, 2050. 2053 BGB). ${ }^{71}$ Insoweit hätte von einer nicht unwesentlichen Erweiterung der Testierfreiheit die Rede sein können; doch wurde dieses Vorhaben, angeblich aus Vertrauensschutzgründen 72 nicht Gesetz.

Bezüglich der folgenschwereren und konfliktträchtigeren Frage nach dem erblasserseits unverfügbaren Kreis der pflichtteilsberechtigten Personen bringt die Neuregelung aber eher eine Einschränkung der Testierfreiheit mit sich, allerdings verbunden mit einem Gewinn an Rechtssicherheit. Trotz der Zunahme und Intensivierung familiärer Entfremdungskonstellationen (ohne kriminelle Komponenten), gerade zwischen Erblassereltern und Abkömmlingen, gibt es auch weiterhin - anders als etwa in Österreich - weder die Möglichkeit zur Reduktion des Pflichtteils noch eine Ausdehnung der Pflichtteilsentziehungsgründe. Im Gegenteil: der Gesetzgeber steuert m. E. einer Erosion der Familiensolidarität in diesem zentralen Bereich des Erbrechts sogar entgegen, indem die „Unterhaltsersatzfunktion“ des Pflichtteils in subtiler Weise eher gestärkt als geschwächt wird. Denn die Generalklausel für die Entziehung des Pflichtteils vom „ehrlosen oder unsittlichen Lebenswandel“ ( $§ 2333$ Nr. 5 BGB a. F.) wurde ersetzt durch die präzisierende Verengung auf eine rechtskräftige Verurteilung zu einer Freiheitsstrafe von mindestens einem Jahr ohne Bewährung 73 und die darauf beruhende Unzumutbarkeit der Nachlassteilhabe für den Erblasser (§ 2333 Abs. 1 Nr. 4 BGB). Prostitution, fortgesetzter Ehebruch, hartnäckige Arbeitsscheu, Verschwendungs-, Spiel-, Alkoholund Drogensucht sowie sonstiges Fehlverhalten, was sich ja gerade auch manche „Kun-

68 Zur mehrfachen Befassung allein des Deutschen Juristentags zuletzt Martiny, Dieter, Gutachten A 64. DJT 2002, A 61 f. m. Nw. zum 14. DJT 1878 und zum 49. DJT 1972.

69 Gesetz zur Änderung des Erb- und Verjährungsrechts, BGBl 2009 I, S. 3142; zuvor Entwurf der Bundesregierung v. 30.1.2008, BT-Drs. 16/8954.

70 Zur verfassungsrechtlichen Garantie der Nachlassteilhabe von Abkömmlingen BVerfG v. 19.4.2005, NJW 2005, S. 1561.

71 Näher Muscheler, Karlheinz, Die geplanten Änderungen im Erbrecht, Verjährungsrecht und Nachlassverfahrensrecht, ZEV 2008, S. 105, 108; Keim, Christopher, Die Reform des Erb- und Verjährungsrechts und ihre Auswirkungen auf die Gestaltungspraxis, ZEV 2008, S. 161, 163 f.; Langenfeld, Gerrit, Das Gesetz zur Änderung des Erb- und Verjährungsrechts - Inhalt und Praxisfolgen, NJW 2009, S. 3121, 3124.

72 Langenfeld (Fußn. 71), NJW 2009, S. 3124 m. Hinw. auf BT-Drs. 16/13543, S. 20.

73 Zur groben Orientierung: mit dem Strafmindestmaß von einem Jahr wird generell die Grenze zwischen Vergehen und Verbrechen markiert (§ 12 Abs. 1 StGB). 
den“ von Sozialleistungsträgern zuschulden kommen lassen, wird somit künftig keine unmittelbare Rolle mehr spielen. Infolgedessen werden die meisten Erblasser mit solcherart belasteten Familienbeziehungen sich künftig schon den Gedanken an eine Pflichtteilsentziehung aus dem Kopf schlagen müssen.

Man mag in derart veranlassten Vermögens(ver)teilungen bloße Nebenwirkungen und günstige Reflexe von geringer sozialfiskalischer Erheblichkeit sehen. Mir scheint indes, dass sie in der budgetären Gesamtbetrachtung durchaus staatsentlastend zu Buche schlagen und deshalb wohl auch nicht ganz ohne darauf gerichtete legislatorische Absicht beibehalten bzw. gestaltet werden.

2. Eindeutig intentionales Vorgehen des Gesetzgebers zur Eindämmung öffentlicher Lasten ist hingegen im Pflegesektor zu beobachten. Im zeitlichen und zweifellos auch funktionalen Zusammenhang mit der 2008 durchgeführten Reform der Pflegeversicherung ${ }^{74}$ sollten auch markante privatrechtliche Anreize und Hilfen zur Übernahme von Angehörigenpflege normiert werden.

Neben neuen arbeitsrechtlichen Freistellungsansprüchen für Pflege und Pflegeorganisation im Pflegezeitgesetz ${ }^{75}$ versuchte der zunächst vorgelegte Erbrechtsänderungsentwurf mit einer neuen Bestimmung, § 2057b BGB-E ${ }^{76}$, zielgerichtet das private Erbrecht weit stärker, als es bisher schon durch $\S 2057$ a Abs 1 S. 2 BGB geschah (und i. W. auch fortan geschieht) in den Dienst öffentlicher Interessen zu stellen. Der verteilende Zugriff auf die Nachlassmasse sollte beträchtlich ausgedehnt werden, indem für die Pflege des Erblassers durch eine (oder mehrere) Personen aus dem Kreis aller gesetzlichen Erben künftig eine praktikablere Ausgestaltung und vor allem eine wertmäBig ergiebigere Bemessung des Pflegeausgleichs angesetzt wurde. ${ }^{77}$ Bemerkenswert war der vorgesehene Umfang: der den Erblasser pflegende gesetzliche Erbe sollte (mangels anderweitiger Honorierung) aus dem Nachlass zusätzlich zu seinem Erbteil den Wert der Pflegesachleistung nach § 36 SGB XI erhalten. Da dieser Wert das Pflegegeld für die selbstbeschaffte Pflegehilfe nach § 37 SGB XI etwa um das Doppelte übersteigt, liegt das Ziel einer nennenswerten Anreizwirkung zur Übernahme von Angehörigenpflege hier offen zutage. In der öffentlichen Berichterstattung über die anstehende Erbrechtsreform hatte sich dieser Neuerungsvorschlag zu einem bislang versteckten und schwer handhabbaren Erbrechtsinstrument ganz in den Vordergrund geschoben und wurde allenthalben als Fortschritt an privater Verteilungsgerechtigkeit gutgeheißen.

74 Pflege-Weiterentwicklungsgesetz v. 28.5.2008, BGB1. I S.874; Gesetzentwurf der Bundesregierung v. 7.12.2007, BT-Drs. $16 / 7439$.

75 Art. 3 des Gesetzes (Fußn. 69).

76 BT-Drs. 16/8695.

77 Näher Köbl, Ursula, Die Neuordnung des erbrechtlichen „Pflegeausgleichs“ (§ 2057a BGB-§ 2057b E-BGB), in: Helms/Zeppernick (Hrsg.), Lebendiges Familienrecht, Festschrift für Rainer Frank zum 70. Geburtstag am 14.7.2008, Frankfurt a.M. 2008, S. 159, m. Nw. zu zahlreichen verbandlichen Stellungnahmen. 
In der rechtspolitischen und rechtswissenschaftlichen Fachdiskussion wurden zwar im einzelnen differenzierte Meinungen vertreten, vor allem aber zur Erstreckung solcherart Honorierung von Pflegeleistungen auch auf die Nachlassverteilung bei gewillkürter Erbfolge gedrängt, ${ }^{78}$ sei es durch Einführung entweder eines gesetzlichen Vermächtnisses ${ }^{79}$ oder einer gesetzlichen Nachlassverbindlichkeit. ${ }^{80}$ Auch mir scheint dies sowohl unter dem privatrechtlichen wie auch dem sozialpolitischen Aspekt als im Ansatz gerechtfertigt. Der damit verbundene Eingriff in die Testierfreiheit wöge bei weitem leichter als etwa ein öffentlich-sozialrechtlicher Regress gegen den Nachlass wegen sehr hohen Pflegeaufwandes, wozu Hänlein und Michaelis vor einigen Jahren ein fundiertes Konzept vorgelegt haben. 81 Verfassungsrechtlich wäre im Prinzip ein solcher Regress ohne weiteres haltbar, da die Pflegeversicherungslösung als solche keineswegs sozialstaatlich zwingend geboten ist, und zudem das Volumen der rückgriffsfreien Leistungen der Pflegeversicherung ja erheblich höher als das Beitragsaufkommen eines gesamten Erwerbslebens angesetzt werden könnte. ${ }^{82}$

Dergleichen Überlegungen sind hier nicht weiter zu verfolgen, denn der skizzierte Entwurf eines erweiterten erbrechtlichen Pflegeausgleichs wurde schließlich wieder fallengelassen, mit der Begründung, dass ,eine Erweiterung des Kreises der Anspruchsberechtigten ... zu einer Vielzahl von Folgeproblemen und Abgrenzungsfragen" führe. ${ }^{83}$ Es bleibt zunächst bei $\S 2057$ a Abs. 1 S.2 BGB, lediglich mit der allseits gebilligten Streichung der (wohl sogar verfassungswidrigen) Tatbestandsvoraussetzung ,unter Verzicht auf berufliches Einkommen“. Ein größerer Verteilungszugriff auf Privatvermögen zur Entlastung der Pflegeversicherung findet einstweilen nicht statt, mit einer Zunahme der Berufungen auf $\S 2057$ a BGB bei Erbauseinandersetzungen dürfte indessen zu rechnen sein.

78 Aus naheliegenden finanziellen Gründen die Bundesländer BR-Drs. 96/08; ferner die im Internet abrufbaren Stellungnahmen der Bundesrechtsanwaltskammer und des Deutschen Juristinnenbundes; zu Durchführungsproblemen Köbl, in: Helms/Zeppernick (Hrsg.), Lebendiges Familienrecht (Fußn. 77), S. 177.

79 Dafür insbesondere Otte, Gerhard, Bessere Honorierung von Pflegeleistungen - Plädoyer für eine Vermächtnislösung, ZEV 2008, S. 260 (bezüglich des Umfangs aber nur für eine Bemessung nach $\S 37$ SGB XI).

80 Dafür insbesondere Windel, Peter A., Wie ist die häusliche Pflege aus dem Nachlass zu honorieren?, ZEV 2008, S. 305. Ähnlich wie Otte (Fußn. 79), ZEV 2008, S. 260, präferiert auch von Krug, Walter, Die Berücksichtigung lebzeitiger Leistungen einzelner Miterben in der Erbteilung, ZFE 2008, S. 324; (für eine Bemessung nur nach $\S 37$ SBG XI und nicht $\S 36$ SGB XI auch Windel, a. a. O. S. 307).

81 Hänlein, Andreas/Michaelis, Jochen, Ersatzpflicht des Erben für Aufwand der Pflegeversicherung ein Vorschlag zur Reform der Pflegeversicherung, NDV 2006, S. 250.

82 Dieser und andere Gesichtspunkte zum Reformvorschlag bei Udsching, Peter, Versorgung bei Pflegebedürftigkeit im Alter - Interdependenzen von Renten- und Pflegeversicherung, in: Becker (Hrsg.), Alterssicherung in Deutschland, Festschrift für Franz Ruland zum 65. Geburtstag, BadenBaden 2007, S. 559, $570 \mathrm{f}$.

83 BT - Drs. J16 / 13543, S. 12. 
3. Ein sozialhilferechtlich motivierter Zugriff auf Nachlassvermögen im Zusammenhang mit sog. Behindertentestamenten ${ }^{84}$ wurde bisher durch die höchstrichterliche $\mathrm{Zi}$ vilrechtsprechung abgewehrt. Unter Behindertentestament versteht man, etwas verkürzt ausgedrückt, eine kautelarjuristisch ausgeklügelte Erbfolgegestaltung von Eltern eines behinderten, auf Kosten der Sozialhilfe lebenslang stationär unterzubringenden Kindes, bei der durch die Kombination von dessen bloßer nichtbefreiter Vorerbenstellung mit lebenslanger Testamentsvollstreckung der Wert des diesem Kind an sich zustehenden Pflichtteils, selbst nach seinem Tod, vor dem Regress des Sozialhilfeträgers endgültig bewahrt wird. In solchen Fällen, im Ergebnis mit dem BGH, einen Verstoß der Verfügung von Todes wegen gegen $\S 138$ Abs. 1 BGB abzulehnen, erscheint mir sozialstaatlich allenfalls bei vergleichsweise bescheidenen Nachlasswerten hinnehmbar, ${ }^{85}$ etwa dann, wenn auch bei Heranziehung des Pflichtteils die stationären Unterbringungskosten nur für eine verhältnismäßig kurze Zeit gedeckt wären.

Die strenge Subsidiarität der Leistungen von Grundsicherung und Sozialhilfe (SGB II und XII) sowie eine zunehmende Polarisierung der Vermögensverhältnisse werfen noch mancherlei Fragen nach den Grenzen zwischen gesamtgesellschaftlicher und familiärer Solidarität auf. Genannt sei hier nur noch der Versuch zur Entwicklung eines „Bedürftigentestaments“, welches ähnlich wie das Behindertentestament die Substanz eines Nachlasses oder Erbteils vor der Anrechnung auf Leistungen nach SGB II und SGB XII bewahren soll. ${ }^{86}$

Es gilt in diesem Bereich noch Fragen zu vertiefen, die auch zu der von Zacher scharfsichtig gekennzeichneten und sozialpolitisch marginalisierten „Problematik des

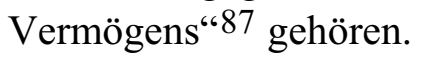

\section{Schlussbemerkung: Unvermeidlichkeit von Zielkonflikten}

Ergibt unser Durchgang durch die neueren Rechtsentwicklungen auf dem weiten Feld der Familiensolidarität auch kein völlig einheitliches und geschlossenes Bild, was das inmitteliegende Potential zur Entlastung öffentlicher Haushalte anbelangt - vor al-

84 Grundsätzlich zur Problematik BGH v. 21.3.1990, Z 111, 36; BGH v. 20.10.1993, Z 123, 368; Frank, Rainer, Erbrecht, 4. Aufl. München 2007, § 3 Rdnr. 14 ff.; Köbl, Ursula, Schranken der Privatautonomie aufgrund Sozialhilferechts, ZfSH/SGB 1990, S. 449.

85 Sehr krit. Raiser, Günther H., Sittenwidrige Behindertentestamente und unerträgliche Belastung der Allgemeinheit, MDR 1995, S. 237; ähnlich Köbl (Fußn. 84), ZfSH/SGB 1990, S. 465.

86 Dazu Litzenburger, Wolfgang, Das Bedürftigentestament - Erbfolgegestaltung zu Gunsten von Langzeitarbeitslosen (Hartz-IV-Empfängern), ZEV 2009, S. 278. Im weiteren Umkreis zur vorweggenommenen Erbfolge: die Verneinung der Sittenwidrigkeit des Ausschlusses von Versorgungsleistungen im Fall des Pflegeheimaufenthalts (BGH, ZEV 2009, S. 254).

87 Zacher, in: Fiedler (Fußn. 1), S. 955, 980; ders., Abhandlungen (Fußn. 1), S. 555, 579 f. 
lem die Schaffung der Grundsicherung im Alter und bei voller Erwerbsminderung wie auch die verstärkte Übernahme von Behindertenunterhaltslasten fallen markant heraus so darf doch insgesamt von einer Art „,impliziter Sozialpolitik“ die Rede sein, die vermehrt auf den Einsatz privater Ressourcen setzt.

Abschließend sei stichwortartig auf die in einer jeden, öffentlichen wie privaten, Familienpolitik angelegten Zielkonflikte hingewiesen.

Da ist zunächst - bedauerlicherweise ein Grunddilemma des Sozialrechts - die Steigerung der individuellen Sorglosigkeit und unvernünftigen Risikobereitschaft, etwa zur Eingehung erhöhter, ja extremer familiärer Risiken durch Schwangerschaften bei fehlender oder labiler Partnerbindung oder umgekehrt zur übereilten Auflösung solcher Bindungen trotz gemeinsamer Kinder.

Ein Weiteres: die Ausschöpfung familialen Sorgepotentials für Kinder und pflegebedürftige Angehörige behindert die faktische Gleichstellung der Geschlechter sowie die langzeitliche Vereinbarkeit von Familie und Erwerbstätigkeit vor allem für Frauen. Der Schutz und das Ansehen der Ehe erleiden infolge der Angleichung der Rechtsstellung der ehelichen und der nichtehelichen Kinder zwangsläufig einen dramatischen Niedergang. ${ }^{88}$

Damit soll gesagt sein: Forderungen nach strikter staatlicher Neutralität gegenüber dem breiten Spektrum von gelebten oder erstrebten Familienmodellen unserer Gesellschaft, um die volle Wahlfreiheit der Menschen zu gewährleisten, sind unrealistisch. Selbstverständlich werden jeweils für bestimmte familiäre Lebensmuster durch einzelne Instrumente des Familien- und Erbrechts, des Arbeits-, Bildungs-, Steuer- und Sozialrechts u. s. w. mehr Anreize gesetzt als für andere. Eben das tut der Staat, wenn er etwa Eheleuten und Familien Steuervergünstigungen einräumt oder wenn er nur für ein Jahr Elterngeld mit Lohnersatzcharakter gewährt (mit zwei Zusatzmonaten für die Väter) und daneben die frühkindliche Betreuung außerhalb der eigenen Familie zu gewährleisten sucht. ${ }^{89}$ Mehr als günstige Rahmenbedingungen für Elternschaft und wirtschaftliche Selbstständigkeit der Individuen zu schaffen, lassen die Menschenwürde- und die Freiheitsgarantien unserer Verfassung gar nicht zu. Der gesellschaftliche Wandel, die oben angerissenen Zielkonflikte und die kurzphasigen Fokussierungen des politischen Prozesses werden stets neue Reformvorstellungen hervorbringen.

Den Solidaritätsgedanken noch einmal aufgreifend sei eine letzte Bemerkung zur sozialstaatsentlastenden, allgemein mitmenschlichen Solidarität angefügt: Fortschritte beträchtlichen Ausmaßes könnten künftig erzielt werden, wenn jenseits der familiär

88 Vgl. Schwab (Fußn. 1), FamRZ 2007, S. 4; ders., FAZ v. 23.11.2006, Nr. 273, S. 8; Born (Fußn. 32), NJW 2008, S. 8.

89 Wenig sinnvoll daher z.B. die Stellungnahme des Podiums (Vertreter von Sozialpartnern und Sozialversicherung) in GVG, Informationsdienst Nr. 318 (Fußn. 4): „Eine direkte staatliche Förderung mit dem Ziel einer erhöhten Geburtenrate ist im Interesse der Entscheidungsfreiheit des Einzelnen und der Familie nicht wünschenswert.“ 
partnerschaftlichen und intergenerationellen Beziehungen und weit über diese hinaus, ${ }^{90}$ namentlich die Potentiale des rüstigen Alters, aber auch die von Jugendlichen und jedweder sonstigen Personenkreise, sich für den Ausbau eines breit gefächerten bürgerschaftlichen Engagements aktivieren ließen. Ministerielle Aufrufe, organisatorische und finanzielle Anschubleistungen, wie beispielsweise zum „Freiwilligendienst aller Generationen“, 91 gehen jedenfalls seit einiger Zeit mannigfaltig ins Land. ${ }^{92}$

90 Allgemein zur überfamiliär intergenerationellen Solidarität Zacher, in: Lorenz (Hrsg.), Festschrift für Andreas Heldrich (Fußn. 11), S. 1216 f.

91 BMFSFJ, Pressemitteilung Nr. 345/2008 v. 13.11.2008.

92 Vgl. nur in dichter Folge jeweils von BMFSFJ Internetredaktion: u. a. am 1.12.2008 zum bundesweiten Projekt „Migrantenorganisationen als Träger von Freiwilligendiensten”; am 15.1.2008 zum Internetportal „Neue Wege für Jungs”; am 2.2.2009 zum Programm „Aktiv im Alter” - zweite Ausschreibungsrunde; am 5.2.2009 zum Wettbewerb „Innovative Wohngemeinschaften”; am 2.12.2009 (Amtsantritt der neuen Familienministerin): „,...die Potentiale nutzen, die das Altern unserer Gesellschaft mit sich bringt“; am 18.12.2009 zum Projekt „Wohnen Plus - Alte, Alleinerziehende und Kinder“; Koalitionsvertrag zwischen CDU,CSU und FDP, Okt. 2009, S. 59. 


\section{Diskussionsbericht}

I. Die Diskussion befasste sich mit den Funktionen und Perspektiven von Sozialleistungen zur Familienförderung sowie mit den Unklarheiten hinsichtlich des soziologischempirischen Konstrukts dessen, was als Familie verstanden wird angesichts des Funktionswandels der Familie und seiner rechtsdogmatischen Implikationen. Kontroverse Aspekte der Diskussion waren die Steuerungsfunktion der Familienförderung, das Verhältnis von Familien- und Sozialrecht sowie die Bedeutung von Kinderrechten zwischen elterlichem Erziehungsauftrag und staatlicher Förderpflicht.

II. Es herrschte Konsens darüber, dass Familienförderung heute eine Vielzahl von Funktionen erfüllt, um der Pluralität von Familienformen Rechnung zu tragen. Dabei wurden zwei rechtsdogmatische Begründungen gegenübergestellt: So wurde betont, dass das Sozialrecht in Bezug auf Familien nicht nur den Zweck verfolge, Gleichheit zu generieren und potentielle Beeinträchtigungen der Gleichheit bei Eltern und Erziehenden zu minimieren. Sozialrecht habe auch die Aufgabe, Freiheit zu sichern und überhaupt erst Handlungsfreiräume für Familien zu eröffnen, damit sie die Möglichkeiten des modernen Familienrechts nutzen können, das durch eine im Vergleich zu früher größere Autonomie in der Gestaltung der familiären Beziehungen geprägt ist. Gleichzeitig wurde auf die bestehenden Unklarheiten in der Mikrostruktur der Familienförderung (etwa bei der Frage, wer durch das deutsche Kindergeld genau gefördert werden soll) hingewiesen, die eine rechtsdogmatisch schlüssige Annäherung an dieses Teilgebiet des Sozialrechts erschweren.

Vor dem Hintergrund der zu Art. 6 Grundgesetz entwickelten Dogmatik, die neben dem Aspekt der Förderung von Ehe und Familie als Institution auch den freiheitsrechtlichen Charakter von Art. 6 GG hervorhebt, wurde dafür plädiert, dass Familienförderung heute sich nicht auf Leistungen beschränken dürfe, die an ein bestimmtes Rollenmuster anknüpfen, sondern dass grundsätzlich alle familiären Rollengestaltungen zu fördern seien. Aus diesem Grund sei die Ausgestaltung des neuen Elterngeldes problematisch, da es nur bestimmte Rollenmuster unterstütze.

Nachdrücklich plädiert wurde außerdem für eine explizite Perspektiverweiterung der Familienförderung auf die Kinderperspektive, die wiederum den Fokus auf die sozialen Dienstleistungen richte. Dabei wurde einerseits unter Hinweis auf die gestiegene Kinderarmut die Frage nach der Kindergerechtigkeit - und damit der Chancengleichheit aufgeworfen, wenn heute die Kinder- und Jugendbehörden bis zu 18 \% Kinderarmut zu verwalten haben. Mit diesem empirischen Befund stelle sich auch die Frage nach der Umsetzung von Recht und den realen Möglichkeiten, Kindern und Eltern in Not die nach Gleichheitsmaßstäben gebotene Hilfe zu gewähren. Die Verlagerung der Gesetzgebungskompetenz auf die (finanzschwächeren) Länder wurde ebenfalls unter gleichheitspolitischen Aspekten kritisiert. 